\title{
Clinical, genomics and networking analyses of a high-altitude native American Ecuadorian patient with congenital insensitivity to pain with anhidrosis: a case report
}

Andrés López-Cortés ${ }^{1,2^{*}} \mathbb{D}$, Ana Karina Zambrano ${ }^{1 \dagger}$, Patricia Guevara-Ramírez ${ }^{1 \dagger}$, Byron Albuja Echeverría ${ }^{3 \dagger}$, Santiago Guerrero ${ }^{1 \dagger}$, Eliana Cabascango ${ }^{4}$, Andy Pérez-Villa', Isaac Armendáriz-Castillo ${ }^{1}$,

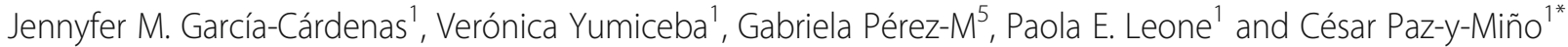

\begin{abstract}
Background: Congenital insensitivity to pain with anhidrosis (CIPA) is an extremely rare autosomal recessive disorder characterized by insensitivity to pain, inability to sweat and intellectual disability. CIPA is caused by mutations in the neurotrophic tyrosine kinase receptor type 1 gene (NTRK1) that encodes the high-affinity receptor of nerve growth factor (NGF).

Case presentation: Here, we present clinical and molecular findings in a 9-year-old girl with CIPA. The highaltitude indigenous Ecuadorian patient presented several health problems such as anhidrosis, bone fractures, selfmutilation, osteochondroma, intellectual disability and Riga-Fede disease. After the mutational analysis of NTRK1, the patient showed a clearly autosomal recessive inheritance pattern with the pathogenic mutation rs763758904 (Arg602*) and the second missense mutation rs80356677 (Asp674Tyr). Additionally, the genomic analysis showed 69 pathogenic and/or likely pathogenic variants in 46 genes possibly related to phenotypic heterogeneity, including the rs 324420 variant in the FAAH gene. The gene ontology enrichment analysis showed 28 mutated genes involved in several biological processes. As a novel contribution, the protein-protein interaction network analysis showed that NTRK1, SPTBN2 and GRM6 interact with several proteins of the pain matrix involved in the response to stimulus and nervous system development.
\end{abstract}

Conclusions: This is the first study that associates clinical, genomics and networking analyses in a Native American patient with consanguinity background in order to better understand CIPA pathogenesis.

Keywords: CIPA, Native American, Ecuadorian, NTRK1, Genomics analysis

\footnotetext{
*Correspondence: aalc84@gmail.com; cesar.pazymino@ute.edu.ec

${ }^{\dagger}$ Andrés López-Cortés, Ana Karina Zambrano, Patricia Guevara-Ramírez, Byron

Albuja Echeverría and Santiago Guerrero contributed equally to this work.

'Centro de Investigación Genética y Genómica. Facultad de Ciencias de la

Salud Eugenio Espejo, Universidad UTE, Mariscal Sucre Avenue, 170129 Quito,

Ecuador

Full list of author information is available at the end of the article
}

C C The Author(s). 2020 Open Access This article is licensed under a Creative Commons Attribution 4.0 International License, which permits use, sharing, adaptation, distribution and reproduction in any medium or format, as long as you give appropriate credit to the original author(s) and the source, provide a link to the Creative Commons licence, and indicate if changes were made. The images or other third party material in this article are included in the article's Creative Commons licence, unless indicated otherwise in a credit line to the material. If material is not included in the article's Creative Commons licence and your intended use is not permitted by statutory regulation or exceeds the permitted use, you will need to obtain permission directly from the copyright holder. To view a copy of this licence, visit http://creativecommons.org/licenses/by/4.0/. The Creative Commons Public Domain Dedication waiver (http://creativecommons.org/publicdomain/zero/1.0/) applies to the data made available in this article, unless otherwise stated in a credit line to the data. 


\section{Background}

Congenital insensitivity to pain with anhidrosis (CIPA), also known as hereditary sensory and autonomic neuropathy Type IV (HSAN-IV) (OMIM \#256800), is an extremely rare autosomal recessive disorder characterized by axonal atrophy affecting the sensory and autonomic neurons [1, 2]. HSAN-IV is characterized by recurrent episodes of unexplained fever, self-mutilating behavior, anhidrosis, absence of reaction to noxious stimuli, intellectual disability [3], humoral immunodeficiency [4], palmoplantar keratoderma $[5,6]$, and early onset renal disease [7]. This condition occurs with an incidence of 1 in 125 million newborns [8].

Brain regions with pain perception are complex and have been best described as a pain matrix [9, 10]. According to Foulkes et al (2008), it consists of four phases in which different genes/proteins are involved [10]. Nerves inside the skin have the ability to transmit the sensation of heat, cold and mechanical stimulation; $\mathrm{Na}^{+}$ and $\mathrm{K}^{+}$channels drive nerve stimuli; the synaptic transmission occurs in the spinal cord via neurotransmitter receptors and $\mathrm{Ca}^{2+}$ channels; lastly, central, peripheral and microglia modulation occurs in brain [10]. Nevertheless, patients with CIPA may present genetic alterations causing functional disruption in one of these pain matrix phases.

Only some hundred of cases of CIPA have been reported worldwide $[8,11]$. The first reference to a similar pathology was mentioned by Dearborn in 1932 [12], and it was published in 1963 by Swanson [13]. Tunçbilek et al (2005) determined three clinical representative findings: insensitivity to pain, inability to sweat and intellectual disability [14]. Indo et al (1996) associated CIPA pathogenesis with genetic loss-of-function mutations of the NTRK1 (neurotrophic receptor tyrosine kinase 1) gene [15]. Grills and Schuijers (1998) postulated that NGF function disruption also causes an altered process of fracture consolidation [16]. Indo et al (2001) determined that CIPA is not only an autosomal recessive disorder, but also a uniparental disomy [17]. Jarade et al (2002) observed ocular manifestations [18]. Many studies of Weier et al (1995), Miura et al (2000), Indo et al (2001), Mardy et al (2001), Bonkowsky et al (2003) and Lin et al (2010) discovered novel mutations and polymorphisms in NTRK1 causing CIPA [19-23]. Schreiber et al (2005) analyzed insulin-related difficulties [24]. Brandes and Stuth (2006) evaluated anesthetic considerations [25]. Many studies of Tanaka et al (1990), Indo (2002) and Melamed et al (2004) determined that NGF receptor failure causes a deficient development of dorsal root neurons (pain and temperature sensory system) autonomic sympathetic neural system (eccrine sweat glands innervation) [26, 27]. Abdulla et al (2014) observed heterotopic ossification and callus formation following fractures and eventually Charcot's joint [28]. Franco et al (2016) proposed that mutations of NTRK1 generate different levels of cell toxicity, which may provide an explanation of the variable intellectual disability observed in CIPA [29]. Altassan et al (2016) identified novel NTRK1 mutations in CIPA individuals through exome DNA sequencing [1]. Finally, Habib et al (2019) identified a microdeletion and a polymorphism (rs324420) in the $F A A H$ gene with high anandamine concentrations and pain sensitivity [30].

NTRK1, also known as TRKA, encodes the neurotrophic tyrosine kinase-1 receptor, which is autophosphorylated activating various intracellular signaling transduction such as cell survival, growth and differentiation [1, 26, 31]. Additionally, the pain insensitivity is caused by the absence of the NGF-dependent primary afferents, and anhidrosis is explained by the lack of the sympathetic postganglionic neurons [1, 32]. According to the Human Gene Mutation Database and the ClinVar, NTRK1 has $\sim 79$ alterations among single nucleotide polymorphisms (SNPs), insertions and deletions, inherited in an autosomal recessive pattern [33, 34]. Indo et al (1996) has reported for the first time NTRK1 mutations associated with CIPA in an Ecuadorian family [15]. However, this is the first time that clinical, genomics, protein-protein interaction $(\mathrm{PPi})$ networking and gene ontology (GO) enrichment analyses were performed in a high-altitude Native American (indigenous) patient with CIPA disease and family consanguinity background.

\section{Case presentation}

The Human Research Ethics Committee from Universidad San Francisco de Quito (No. 2018-127E) approved all experimental protocols. The methods were carried out in accordance with the relevant guidelines and regulations. Lastly, written informed consent to participate was obtained from all of the participants in this study. In case of CIPA patient, a written informed consent to participate was obtained from their parents.

The case of a 9-year-old girl who was born in the community of Piaba (2418 $\mathrm{m}$ above sea level, MASL), in Cotacachi, located in the north of Ecuador, is presented. She is diagnosed with CIPA, which begins to be suspected after $72 \mathrm{~h}$ of life, where she developed fever of unknown origin; consequently, she was admitted to the hospital, she stayed there for 26 days and she was discharged without specific diagnosis. After 1 month of age, she presented recurring episodes of fever.

When she was 4 months old, she was diagnosed with pneumonia; while she stayed at the hospital, her neurodevelopment was examined by means of the Denver test which provided the following result: unusual for her age. The neurological examination showed generalized hypotonia, active symmetrical movements, incomplete 
cephalic support, absence of pain sensitivity during peripheral line placement, normal deep tendon reflex, and absence of corneal reflex. Additionally, it was observed that there was a $1-\mathrm{cm}$ dermal ulcer, with regular edges on the proximal and distal phalange of the first finger of the right hand, and lesions in healing process on the second finger (Fig. 1a); consequently, with general anesthesia, a skin biopsy of the sternal region was taken to analyze presence or absence of nerve terminals by immunohistochemical staining for S100 protein (Fig. 1b). The negative results showed superficial and deep dermis with some cutaneous appendices constituted by hair follicles and sweat glands that in multiple cuts have no innervation zones, which can be corroborated since the mother presents absence of perspiration.

When she was 16 months old, she was diagnosed with Riga-Fede disease because she presented ulcerative plaques in the oral mucosa and tongue deformities (Fig. 1a). Furthermore, due to previous ulcers, the patient developed osteomyelitis on the distal phalanges of first and second fingers of the left hand (positive culture for Staphylococcus aureus sensitive to cephalexin). At 2.5 years old, and later at 3 years and 2 months old she presented bilateral corneal ulcers of traumatic origin. At 6 years and 4 months old she suffered a tibia fracture caused by falling (Fig. 1c). At 6 years and 7 months she presented a distal tibial fracture without a determined cause. During the consolidation process a mass was found in the fracture area; a biopsy was carried out and osteochondroma was diagnosed. At 8 years and 1 month she broke her left femur because of a fall. Finally, at 8 years and 5 months old she suffered a subtrochanteric fracture of the right femur, requiring surgery (Fig. 1c). As for the family background, a sister of the patient a)
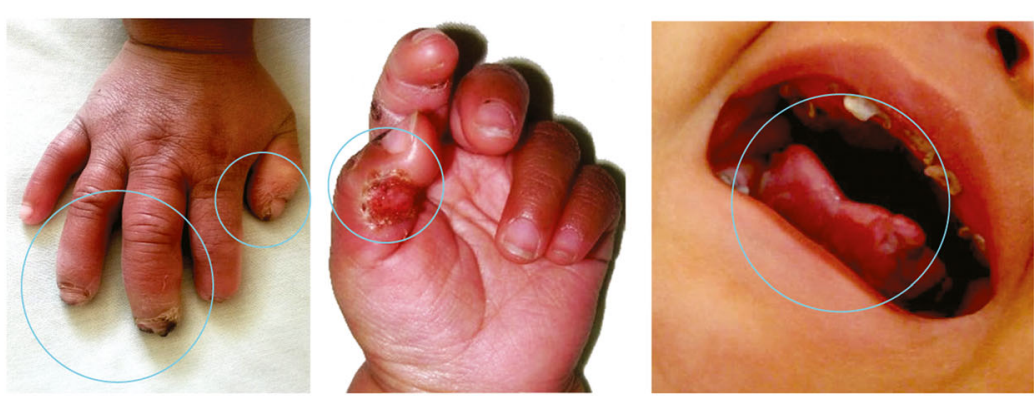

b)

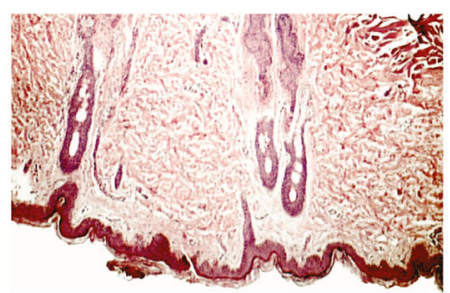

c)

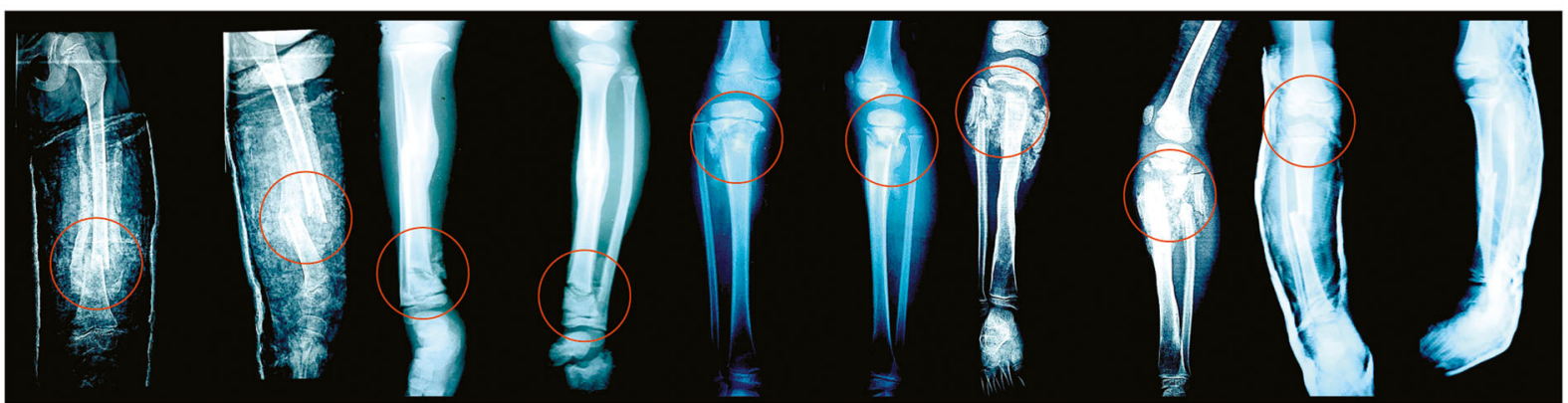

d)

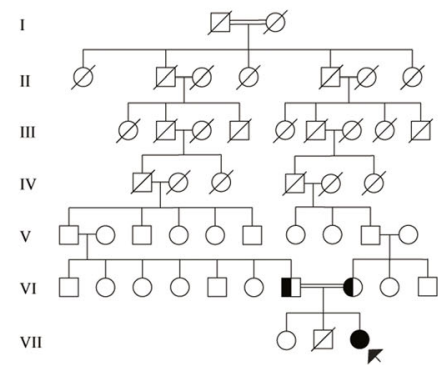

Fig. 1 Clinical features of CIPA patient. a Self-mutilation. b Skin biopsy shows a thin epidermis with hyperkeratosis, there are few sebaceous glands and no nerve terminals are observed. c Several fractures in tibia and femur. d Family genealogical tree 
passed away at 18 months old after developing fever of unknown origin. Figure $1 \mathrm{~d}$ details the genealogical tree, and consanguinity between relatives of both parents. In addition, the patient presented a normal karyotype 46, XX (Figure S1).

Regarding methodology, peripheral blood samples were extracted from CIPA patient and her parents using FTA buffer (GE Healthcare, UK). Genomic DNA was extracted from whole blood using the PureLink Genomic DNA Kit (Invitrogen, Carlsbad, CA), and purified using Amicon Ultra centrifugal filters (Merck, Darmstadt, Germany). Genomic DNA presented a concentration of $45 \mathrm{ng} / \mu \mathrm{L}$ (mother), $27 \mathrm{ng} / \mu \mathrm{L}$ (father) and $36 \mathrm{ng} / \mu \mathrm{L}$ (patient), using a Qubit 4 (Thermo Scientific, Waltham, MA).

Genotyping was performed through PCR reaction of 54 SNPs located into 14 regions of NTRK1 and Sanger sequencing analysis. Table S1 details features of primers and all 54 variants analyzed. Figures S2a and S2b detail the PCR and the sequencing analysis protocols for all genetic variants, respectively. After carrying out the sequencing analysis of the NTRK1 gene, the patient was observed to have the homozygous mutant genotype T/T of the missense mutation rs80356677 (Asp674Tyr). At the nucleotide level there is a change of the guanine (G) to thymine $(\mathrm{T})$ in DNA. Regarding parents, both have the heterozygous genotype $\mathrm{G} / \mathrm{T}$ of the mutation rs80356677 (Figure S2c).

Furthermore, a trio analysis of genomic DNA of mother, father and patient was done by using the TruSight One (TSO) Next-Generation Sequencing (NGS) Panel (Illumina, Inc. San Diego, CA, USA), which includes 125,395 probes targeting a $12-\mathrm{Mb}$ region spanning 62,000 target exons of 4811 genes, and sequenced on the Illumina MiSeq platform. Raw sequence reads were processed and aligned against the human NCBI GRCh37 hg19 reference genome assembly using the BWA software. The 80 -mer probes target libraries with $\sim 500$ bp mean fragment sizes and $\sim 300$ bp insert sizes, enriching a broad footprint of 350-650 based centered symmetrically around the midpoint of the probe. Therefore, in addition to covering the main exon regions, the panels cover exon-flanking regions, which can provide important biological information such as splice sites or regulatory regions. The TSO coverage was $\geq 20 x$ on $95 \%$ of the target regions in the panel, and the TSO full gene list is detailed in the Table S2.

To analyze data generated from targeted sequencing, the BaseSpace Variant Interpreter software (Illumina), the BaseSpace Interpreter (Illumina), Sorting Intolerant From Tolerant (SIFT) (http://sift.bii.a-star.edu.sg/) [35], Polymorphism Phenotyping v2 (PolyPhen-2) (http://genetics.bwh.harvard.edu/pph2/) [36], ClinVar (https:// www.ncbi.nlm.nih.gov/clinvar/) [33], The Human Gene
Mutation Database (http://www.hgmd.cf.ac.uk/ac/index. php) [34], Leiden Open Variation Databases (LOVD) (http://www.lovd.nl/3.0/home) [37], and the Database for Annotation, Visualization and Integrated Discovery (DAVID) (https://david.ncifcrf.gov/) [38] were implemented in the fully detailed bioinformatics pipeline (Fig. 2a).

As results, the total aligned reads was $14,356,459$ (father), 17,975,032 (mother) and 20,225,887 (patient). The percentage of reads passing filter that aligned to the reference was $99.9 \%$ for all samples. The percentage of targets with coverage greater than $20 \mathrm{X}$ was $27.3 \%$ for the father, $30.3 \%$ for the mother, and $30.4 \%$ for the patient with CIPA. Additionally, the analysis of 4811 genes and 18,933 variants was performed in the BaseSpace Variant Interpreter software (Illumina).

After filtering the genomic variants through four steps fully detailed in Fig. 2b, the patient showed a total of 69 pathogenic and/or likely pathogenic variants (Table S3), and 76 variant of uncertain significance (VUS) in 106 genes. The consequence of these 145 variants is fully detailed in Table S4. As results after the DNA genomic analysis, the patient showed a clearly autosomal recessive inheritance pattern with the pathogenic mutation rs763758904 (Arg602\%). The other 69 pathogenic and/or likely pathogenic variants in 46 genes were analyzed to better understand the phenotypic heterogeneity of CIPA.

Consequently, the enrichment analysis of GO terms related to biological processes, cellular components and molecular functions was carried on in 46 genes with pathogenic or likely pathogenic variants using DAVID Bioinformatics Resource in order to better understand the phenotypic heterogeneity of the patient [38]. Only 28 of 46 genes were involved in almost one of the categories showed as a heatmap in Fig. 3. The most significant biological processes (BPs) with a BenjaminiHochberg false discovery rate $(\mathrm{FDR})<0.01$ were muscle contraction, maintenance of gastrointestinal epithelium, reverse cholesterol transport, phospholipid translocation, skeletal muscle contraction, cytoskeleton organization, sarcomere organization and visual perception. The most significant cellular components (CCs) with a FDR $<0.01$ were late endosome, myosin filament, muscle myosin complex, neuronal cell body, photoreceptor disc membrane, integral component o plasma membrane, high-density lipoprotein particle, myofibril and cell junction. In addition, the most significant molecular functions (MFs) with a FDR $<0.01$ were calmodium binding, phospholipid binding, ATP binding, apolipoprotein binding, microfilament motor activity, histone-lysine $\mathrm{N}$-methyltransferase activity and ATPase activity [38] (Fig. 3). The function of all these genes is detailed in the Table S5. 
a)

\begin{tabular}{|c|c|}
\hline BWA software & Analysis of alignment and annotation. \\
\hline BaseSpace Variant Interpreter & Imports variant call files in the VCF file format generated during analysis of sequencing data. \\
\hline BaseSpace Interpreter & Classifies variants according to their clinical significance (i.e. pathogenic, etc). \\
\hline SIFT & Predicts amino acid substitution based on sequence homology and the physical properties. \\
\hline PolyPhen-2 & Predicts impact of amino acid substitutions on the structure and function of proteins. \\
\hline ClinVar & Public archive of reports of the relationships among human variations and phenotypes. \\
\hline Human Gene Mutation & Database of germline mutations associated with human inherited disease. \\
\hline Leiden Open Variation & Provides a flexible tool for gene-centered collection and display of DNA variations. \\
\hline DAVID bioinformatics resource & Enrichment analysis of biological processes, cellular components and molecular functions. \\
\hline
\end{tabular}

b)

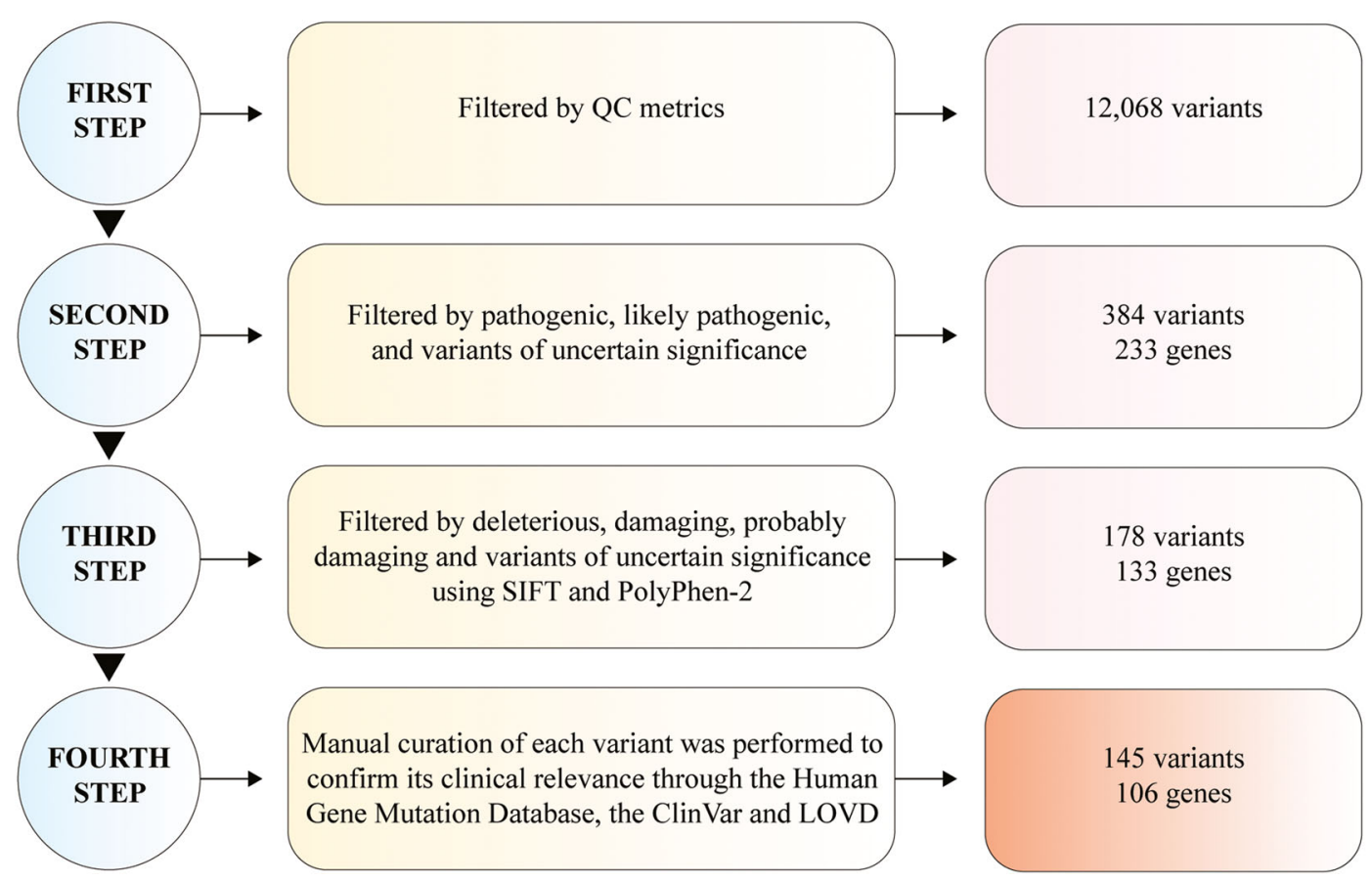

Fig. 2 Next-generation sequencing analysis. a Functions of software and databases used for NGS analysis. b Pipeline of genomic variant analysis

Additionally, a PPi network with a high confidence of $0.7(p<0.001)$ was created using String Database $[39,40]$. This network was made up of known and predicted interactions between proteins with pathogenic and/or likely pathogenic variants and the pain matrix proteins proposed by Foulkes et al (2008) [10], and detailed in Figure S3 and Table S6. As results, three proteins (NTRK1, GRM6, and SPTBN2) with pathogenic and/or likely pathogenic variants interact with several proteins of the pain matrix.
NTRK1 interacts with NGF (peripheral modulation), BDNF (microglia modulation) and TRPV1 (heat transduction). GRM6 interacts with CNR1, OPRD1, OPRK1, OPRM1 (central modulation), CNR2 (peripheral modulation), BDKRB2, BDKRB1 (damage transduction) and CX3CR1 (microglia modulation). Lastly, SPTBN2 interacts with KCNQ3, KCNQ2 (conduction by $\mathrm{K}^{+}$channels), SCN1A, SCN11A, SCN10A, SCN8A and SCN9A (conduction by $\mathrm{Na}^{+}$ channels) (Fig. 4). 


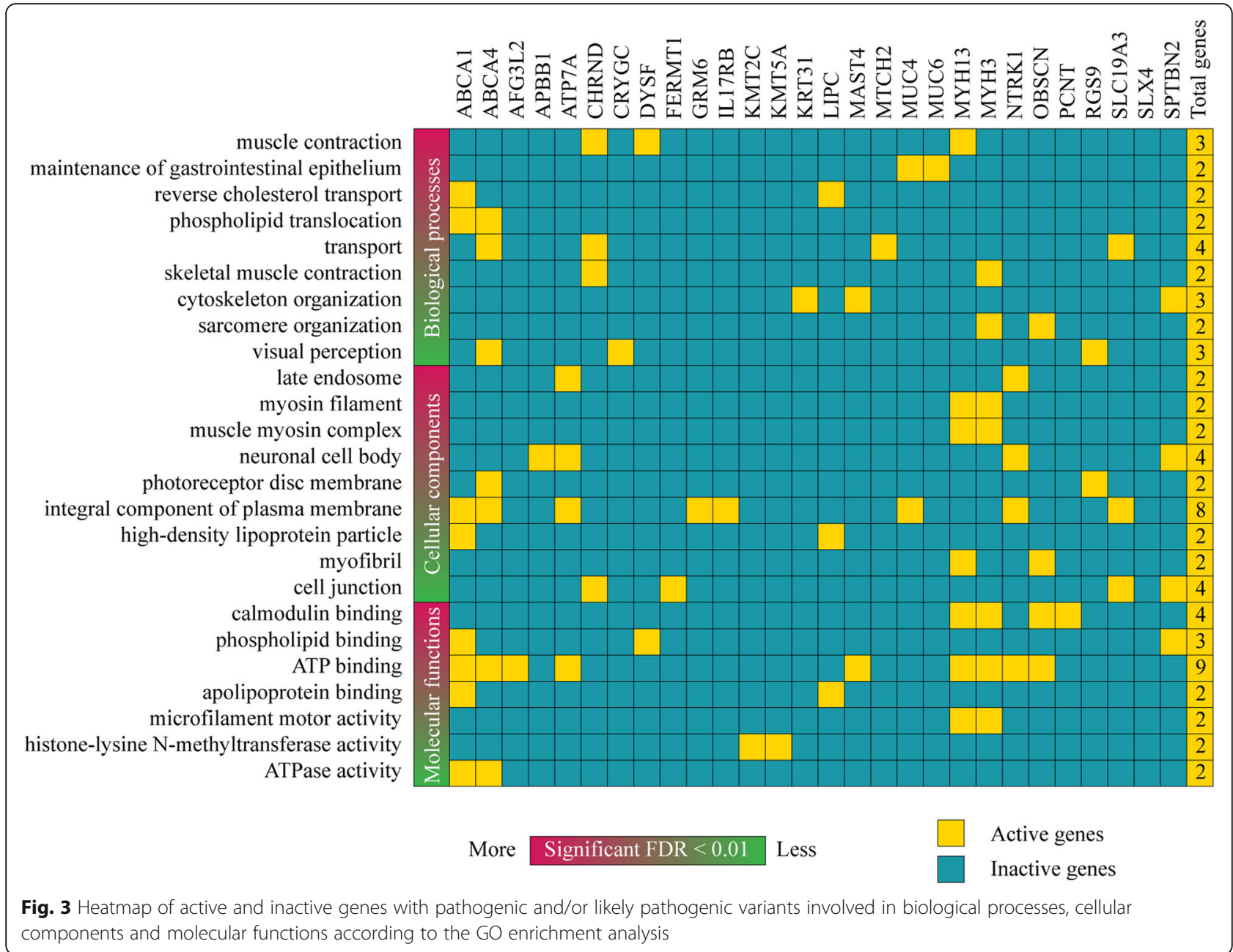

\section{Discussion and conclusions}

In our study, we reported the first case of a Native American patient from a family who lives in a highaltitude indigenous community (2418 MASL) located north of the Ecuadorian highlands.

Nine years of clinical record have shown that the patient with CIPA has presented several health problems such as bone fractures, self-mutilation, osteochondroma, intellectual disability, Riga-Fede disease, ulcers and fever. One of the strengths of this case was the correct followup and adequate intervention and treatment of these health problems, with a positive response and patient tolerance. On the contrary, the biggest limitation of this case is that this autosomal recessive disease has no cure. Regarding patient perspective, parents should share their perspective on the treatments they received.

The NTRK1 pathway is essential for the maintenance of autonomic sympathetic postganglionic neurons because is responsible for innervating skin through sensory axons [1]. In addition, this pathway is involved in regulate vasoconstriction, sweating, endocytosis and vesicular transport in order to promote neural differentiation [41]. The NTRK1 protein receptor is composed of the extracellular, intracellular, tyrosine kinase domains and a carboxyl terminal tail. NGF ligands bind to NTRK1 receptor stimulating autophosphorylation of tyrosine residues and triggering downstream cell signaling [42]. However, NTRK1 mutations lead unfavorable survival of pain receptors and sympathetic ganglion neurons [43]. After carrying out the mutational analysis of NTRK1 and the genomic DNA analysis, the Ecuadorian indigenous patient presented a clearly autosomal recessive inheritance pattern with the pathogenic mutation rs763758904 (Arg602:) and the second missense mutation rs80356677 (Asp674Tyr). rs763758904 (c.1804 C > $\mathrm{T} ; \operatorname{Arg} 602 *)$ is a pathogenic stop gained / splice region variant characterized by the change of Arg to stop codon. Parents presented the heterozygous genotype $(\mathrm{C} /$ $\mathrm{T})$, while the patient presented the homozygous mutant genotype $(\mathrm{T} / \mathrm{T})$. This genetic variant has been reported by Wang et al (2016) in a study on five Chinese children with CIPA [44]. On the other hand, rs80356677 (c.2020 


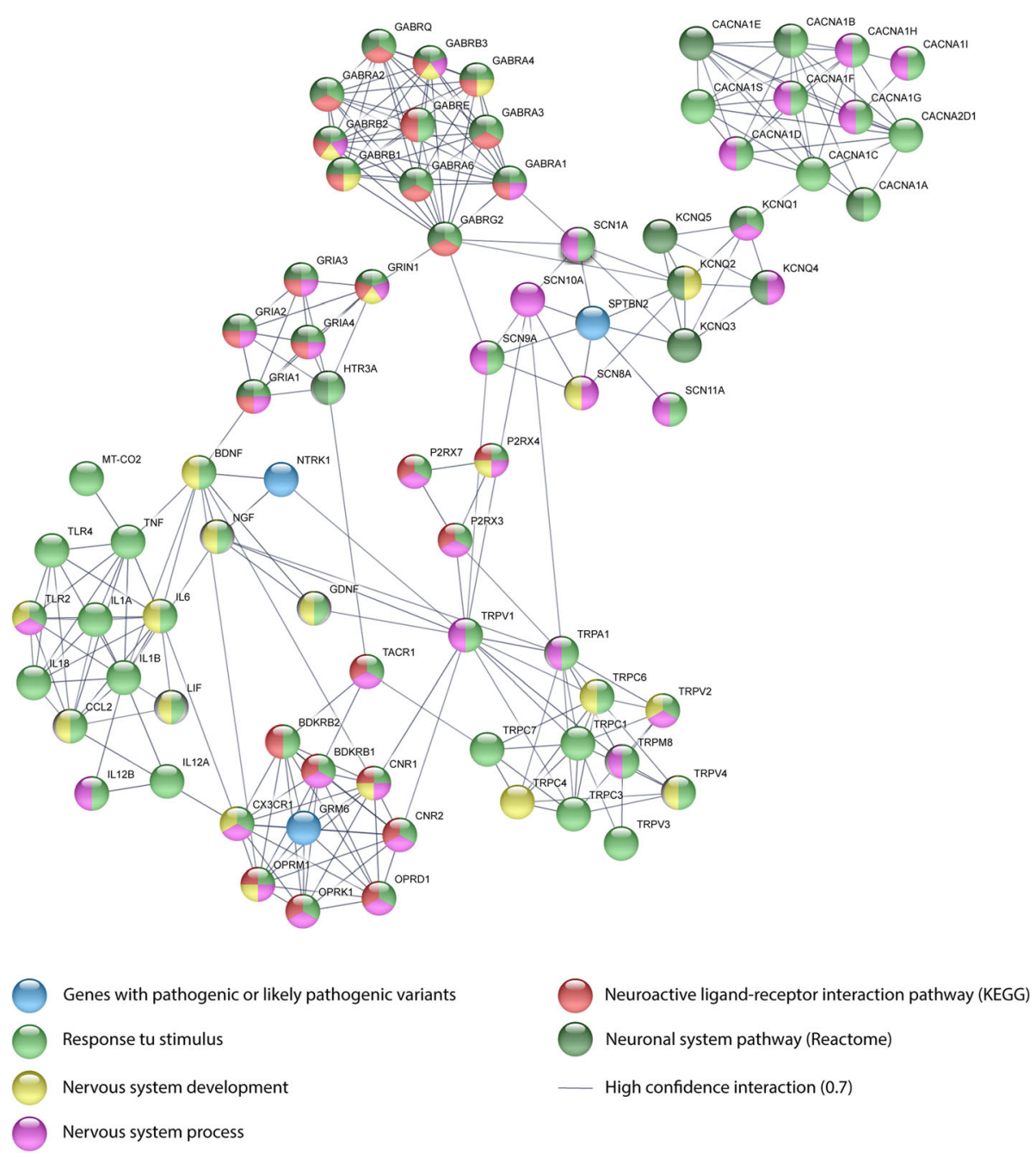

Fig. 4 Protein-protein interaction network between the pain matrix genes and genes with pathogenic or likely pathogenic variants

G > T; Asp674Tyr) is a missense variant characterized by the change of Asp to Tyr in the amino acid 674. Parents presented the heterozygous genotype $(\mathrm{G} / \mathrm{T})$, while the patient presented the homozygous mutant genotype $(\mathrm{T} /$ $\mathrm{T})$. This genetic variant has been reported by Indo et al (2001) [19].

The DNA genomic analysis through NGS showed that the CIPA patient presented 69 pathogenic and/or likely pathogenic variants in 46 genes (Table S3). One of these interesting variants was rs 324420 in the $F A A H$ gene that was identified in a Caucasian female patient with high anandamine concentrations and pain insensitivity [30].

The gene ontology enrichment analysis [38] let us know the possible implication of these 46 genes with the CIPA phenotypic heterogeneity. Only 28 genes were involved in almost one of the categories showed as a heatmap in Fig. 3. The most significant BP was muscle contraction, and transport was the BP with more active genes. The most significant $\mathrm{CC}$ was late endosome, and integral component of plasma membrane was the CC with more active genes. The most significant MF was calmodium binding, and ATP binding was the MF with more active genes. Lastly, the GO terms where NTRK1 was active were late endosome, neural cell body, integral component of plasma membrane and ATP binding.

In regard to the networking analysis showed in Fig. 4, the PPi between proteins with pathogenic and/or likely pathogenic variants and the pain matrix proteins demonstrates that NTRK1 interacts with NGF, BDNF and TRPV1. NGF and BDNF are involved in nervous system development and response to stimulus. TRPV1 is involved in nervous system process and response to stimulus. GRM6 interacts with CNR1, DPRD1, CNR2, BDKRB2, OPRK1, BDKRB1, OPRM1 and CX3CR1. OPRK1, OPRD1, CNR2 and BDKRB1 are involved in nervous system process, response to stimulus and neuroactive ligand-receptor interaction pathway. OPRM1 and CNR1 are involved in nervous system process, response 
to stimulus, neuroactive ligand-receptor interaction pathway and nervous system development. BDKRB2 is involved in neuroactive ligand-receptor interaction pathway and response to stimulus. CX3CR1 is involved in nervous system process, response to stimulus and nervous system development. SPTBN2 interacts with SCN1A, KCNQ3, KCNQ2, SCN11A, SCN10A, SCN8A and SCN9A. SCN11A, SCN1A and SCN9A are involved in nervous system process and response to stimulus. SCN8A is involved in nervous system development and process. SCN10A is involved in nervous system process. $\mathrm{KCNQ} 2$ is involved in nervous system development and the neuronal system pathway. Lastly, KCNQ3 is involved in the neural system pathway $[38,45,46]$.

The gene ontology enrichment analysis and the PPi network can contribute to understand how different genes/proteins with pathogenic variants influence the development of phenotypic patterns, symptoms and complications of CIPA patients worldwide.

In conclusion, we conducted for the first time clinical, genomics, PPi networking and GO enrichment analyses in a high-altitude Native American (indigenous) Ecuadorian patient with CIPA and with family history of consanguinity, whose results were associated with the pain matrix in order to find new proteins related to CIPA pathogenesis and phenotypic heterogeneity.

\section{Supplementary information}

Supplementary information accompanies this paper at https://doi.org/10. 1186/s12920-020-00764-3.

\section{Additional file 1: Figure S1. Karyotype of patient. \\ Additional file 2: Figure S2. Analysis of NTRK1 polymorphisms. a) PCR protocol. b) Sanger sequencing analysis protocol.}

Additional file 3: Figure S3. The pain matrix proteins. We obtained appropriate copyright permission from the corresponding author of the paper (Foulkes et al [10]) to re-design the pain matrix proteins and adapt it as this figure.

Additional file 4: Table S1. Studied mutations of the NTRK1 gene, and their PCR conditions. Table S2. TSO full gene list. Table S3. Pathogenic and likely pathogenic variants in the CIPA patient after DNA genomic analysis. Table S4. Pathogenic variants, probably pathogenic variants and VUS in the CIPA patient after DNA genomic analysis. Table S5. Function of genes with at least one pathogenic or likely pathogenic variant on CIPA patient. Table S6. Full list of the pain matrix genes.

\section{Abbreviations \\ CIPA: Congenital insensitivity to pain with anhidrosis; NTRK1: The neurotrophic tyrosine kinase receptor type 1 gene; NGF: Nerve growth factor; SNP: Single nucleotide polymorphism; PPi: Protein-protein interaction; GO: Gene ontology; MASL: Meters above sea level; dNTP: deoxynucleotide triphosphate; FW: Forward; RV: Reverse; TSO: TruSight One; NGS: Next- generation sequencing; VUS: Variant of uncertain significance; LOVD: Leiden Open Variation Databases; DAVID: The database for annotation, visualization and integrated discovery; BP: Biological process; FDR: False discovery rate; CC: Cellular component; MF: Molecular function}

Acknowledgements

Not Applicable.

\section{Authors' contributions}

ALC conceived the subject, supervised the research project and wrote the manuscript. AKZ and PGR performed the next-generation sequencing anaIysis. BAE, EC, GPM and CPyM interpreted the clinical data and the genomic variants of the patient, provided a genetic counseling to the family, and revised the article critically. SG, IAC and JGC made substantial contribution with bioinformatics analysis. APV, VY and PEL performed the cytogenetic analysis. All authors did data curation and supplementary data. Lastly, all authors made substantive intellectual contributions editing the article before submission, and approved the submitted version.

\section{Funding}

Publication of this article was funded by Universidad UTE. The funding body did not have any role in study design, data collection and analysis, decision to publish, or preparation of the manuscript.

\section{Availability of data and materials}

All data generated during this study are included in this published article including its supplementary tables. DNA sequences are available in NCBI Sequence Read Archive (SRA) with the BioProject accession number PRJNA647341 (https://www.ncbi.nlm.nih.gov/sra/PRJNA647341). Additionally, database used in this study were Sorting Intolerant From Tolerant (SIFT) (http://sift.bii.a-star.edu.sg/), ClinVar (https://www.ncbi.nlm.nih.gov/clinvar/), The Human Gene Mutation Database (http://www.hgmd.cf.ac.uk/ac/index. php), the Database for Annotation, Visualization and Integrated Discovery (DAVID) (https://david.ncifcrf.gov/), Leiden Open Variation Databases (LOVD) (http://www.lovd.nl/3.0/home), Polymorphism Phenotyping v2 (PolyPhen-2) (http://genetics.bwh.harvard.edu/pph2/), and human genome reference GRCh37 hg19 (https://www.ncbi.nlm.nih.gov/assembly/GCF_000001405.13/).

\section{Ethics approval and consent to participate}

This study was approved by the Human Research Ethics Committee from Universidad San Francisco de Quito (No. 2018-127E). Written informed consent to participate was obtained from all of the participants in this study. In case of CIPA patient, a written informed consent to participate was obtained from her parents.

\section{Consent for publication}

Written informed consent was obtained from both of the parents of CIPA patient for publication of this case report. The parents consent for the publication of their medical data and images.

\section{Competing interests}

The authors declare no competing interests.

\section{Author details}

${ }^{1}$ Centro de Investigación Genética y Genómica. Facultad de Ciencias de la Salud Eugenio Espejo, Universidad UTE, Mariscal Sucre Avenue, 170129 Quito, Ecuador. ${ }^{2}$ Latin American Network for Implementation and Validation of Clinical Pharmacogenomics Guidelines (RELIVAF-CYTED), Madrid, Spain. ${ }^{3}$ Hospital San Luis de Otavalo, Ministerio de Salud Pública, Antonio José de Sucre Avenue, 100201 Otavalo, Ecuador. ${ }^{4}$ Sistemas Médicos (SIME), Universidad San Francisco de Quito, Interoceánica Avenue and Chimborazo, 170902 Cumbayá, Ecuador. ${ }^{5}$ Ministerio de Salud Pública, 100117 Ibarra, Ecuador.

Received: 2 January 2020 Accepted: 10 August 2020

Published online: 17 August 2020

\section{References}

1. Altassan R, Al SH, Masoodi TA, Al DH, Khalifa O, Al-Zaidan H, et al. Exome sequencing identifies novel NTRK1 mutations in patients with HSAN-IV phenotype. Am J Med Genet Part A. 2017;173:1009-16.

2. Axelrod FB, Gold-Von SG. Hereditary sensory and autonomic neuropathies: types II, III, and IV. Orphanet J Rare Dis. 2007;2:39.

3. Indo Y. Nerve growth factor and the physiology of??Pain: lessons from congenital insensitivity to pain with anhidrosis. Clin Genet. 2012;82:341-50.

4. Kilic SS, Ozturk R, Sarisozen B, Rotthier A, Baets J, Timmerman V. Humoral immunodeficiency in congenital insensitivity to pain with anhidrosis. Neurogenetics. 2009;10:161-5. 
5. Bonkowsky JL, Johnson J, Carey JC, Smith AG, Swoboda KJ. An infant with primary tooth loss and palmar hyperkeratosis: a novel mutation in the NTRK1 gene causing congenital insensitivity to pain with anhidrosis. Pediatrics. 2003;112:e237-41.

6. Sayyahfar S, Chavoshzadeh Z, Khaledi M, Madadi F, Yeganeh MH, Sawamura $D$, et al. Congenital insensitivity to pain with anhidrosis presenting with palmoplantar keratoderma. Pediatr Dermatol. 2013;30:754-6.

7. Barone R, Lempereur L, Anastasi M, Parano E, Pavone P. Congenital insensitivity to pain with anhidrosis (NTRK1 mutation) and early onset renal disease: clinical report on three sibs with a 25-year follow-up in one of them. Neuropediatrics. 2005;36:270-3.

8. Pérez-López LM, Cabrera-González M, Gutiérrez-de la Iglesia D, Ricart S, Knörr-Giménez G. Update review and clinical presentation in congenital insensitivity to pain and anhidrosis. Case Rep Pediatr. 2015;2015:1-7.

9. Tracey I, Mantyh PW. The Cerebral Signature for Pain Perception and Its Modulation. Neuron. 2007:55:377-91.

10. Foulkes T, Wood JN. Pain Genes. PLoS Genet. 2008:4:e1000086.

11. Gao L, Guo H, Ye N, Bai Y, Liu X, Yu P, et al. Oral and craniofacial manifestations and two novel missense mutations of the NTRK1 gene identified in the patient with congenital insensitivity to pain with Anhidrosis. PLoS One. 2013:8:e66863.

12. Van Ness Dearborn G. A case of congenital general pure analgesia. J Nerv Ment Dis. 1932;75:612-5.

13. Swanson AG. Congenital insensitivity to pain with Anhydrosis: a unique syndrome in two male siblings. Arch Neurol. 1963;8:299-306.

14. Tuncbilek G, Oztekin C, Kayikcioglu A. Calcaneal ulcer in a child with congenital insensitivity to pain syndrome. Scand J Plast Reconstr Surg Hand Surg. 2005;39;180-3.

15. Indo Y, Tsuruta M, Hayashida Y, Karim M, Ohta K, Kawano T, et al. Mutations in the TRKA/NGF receptor gene in patients with congenital insensitivity to pain with anhidrosis. Nat Genet. 1996;13:485-8.

16. Grills BL, Schuijers JA. Immunohistochemical localization of nerve growth factor in fractured and unfractured rat bone. Acta Orthop Scand. 1998;69: 415-9.

17. Indo Y. Molecular basis of congenital insensitivity to pain with anhidrosis (CIPA): mutations and polymorphisms in TRKA (NTRK1) gene encoding the receptor tyrosine kinase for nerve growth factor. Hum Mutat. 2001;18:462-71.

18. Jarade EF, El-Sheikh HF, Tabbara KF. Indolent corneal ulcers in a patient with congenital insensitivity to pain with anhidrosis: a case report and literature review. Eur J Ophthalmol. 2002;12:60-5.

19. Indo Y, Mardy S, Miura Y, Moosa A, Ismail EAR, Toscano E, et al. Congenital insensitivity to pain with anhidrosis (CIPA): novel mutations of the TRKA (NTRK1) gene, a putative uniparental disomy, and a linkage of the mutant TRKA and PKLR genes in a family with CIPA and pyruvate kinase deficiency. Hum Mutat. 2001;18:308-18.

20. Weier HUG, Rhein AP, Shadravan F, Collins C, Polikoff D. Rapid physical mapping of the human trk protooncogene (NTRK1) to human chromosome 1q21-q22 by P1 clone selection, fluorescence in situ hybridization (FISH), and computer-assisted microscopy. Genomics. 1995;26:390-3.

21. Miura Y, Mardy S, Awaya Y, Nihei K, Endo F, Matsuda I, et al. Mutation and polymorphism analysis of the TRKA (NTRK1) gene encoding a high-affinity receptor for nerve growth factor in congenital insensitivity to pain with anhidrosis (CIPA) families. Hum Genet. 2000;106:116-24.

22. Lin YP, Su YN, Weng WC, Lee WT. Novel neurotrophic tyrosine kinase receptor type 1 gene mutation associated with congenital insensitivity to pain with anhidrosis. J Child Neurol. 2010;25:1548-51.

23. Mardy S, Miura Y, Endo F, Matsuda I, Indo Y. Congenital insensitivity to pain with anhidrosis (CIPA): effect of TRKA (NTRK1) missense mutations on autophosphorylation of the receptor tyrosine kinase for nerve growth factor. Hum Mol Genet. 2001;10:179-88.

24. Schreiber R, Levy J, Loewenthal N, Pinsk V, Hershkovitz E. Decreased first phase insulin response in children with congenital insensitivity to pain with anhidrosis. J Pediatr Endocrinol Metab. 2005;18:873-7.

25. Brandes IF, EAE S. Use of BIS monitor in a child with congenital insensitivity to pain with anhidrosis. Paediatr Anaesth. 2006;16:466-70.

26. Indo Y. Genetics of congenital insensitivity to pain with anhidrosis (CIPA) or hereditary sensory and autonomic neuropathy type IV. Clin Auton Res. 2002; $12: 120-3$.

27. Melamed I, Levy J, Parvari R, Gelfand EW. A novel lymphocyte singnaling defect: trk a mutation in the syndrome of congenital insensitivity to pain and anhidrosis (CIPA). J Clin Immunol. 2004;24:441-8.
28. Abdulla M, Khaled SS, Khaled YS, Kapoor H. Congenital insensitivity to pain in a child attending a paediatric fracture clinic. J Pediatr Orthop Part B. 2014;23:406-10.

29. Franco ML, Melero C, Sarasola E, Acebo P, Luque A, Calatayud-Baselga I, et al. Mutations in TrkA causing congenital insensitivity to pain with anhidrosis (CIPA) induce misfolding, aggregation, and mutation-dependent neurodegeneration by dysfunction of the autophagic flux. J Biol Chem. 2016;291:21363-74.

30. Habib AM, Okorokov AL, Hill MN, Bras JT, Lee M-C, Li S, et al. Microdeletion in a FAAH pseudogene identified in a patient with high anandamide concentrationsand pain insensitivity. Br J Anaesth. 2019:123:e249-53.

31. Varma AV, McBride L, Marble M, Tilton A. Congenital insensitivity to pain and anhidrosis: case report and review of findings along neuro-immune axis in the disorder. J Neurol Sci. 2016;370:201-10.

32. Indo Y. Neurobiology of pain, interoception and emotional response: lessons from nerve growth factor-dependent neurons. Eur J Neurosci. 2014; 39:375-91.

33. Landrum MJ, Lee JM, Riley GR, Jang W, Rubinstein WS, Church DM, et al. ClinVar: Public archive of relationships among sequence variation and human phenotype. Nucleic Acids Res. 2014:42:D980-5.

34. Stenson PD, Mort M, Ball EV, Evans K, Hayden M, Heywood S, et al. The human gene mutation database: towards a comprehensive repository of inherited mutation data for medical research, genetic diagnosis and nextgeneration sequencing studies. Hum Genet. 2017;136:665-77.

35. $\mathrm{Ng} \mathrm{PC}$, Henikoff S. SIFT: Predicting amino acid changes that affect protein function. Nucleic Acids Res. 2003;31:3812-4.

36. Adzhubei I, Jordan DM, Sunyaev SR. Predicting functional effect of human missense mutations using PolyPhen-2. Curr Protoc Hum Genet. 2013;7:Unit7. 20.

37. IFAC F, PEM T, GCP S, Celli J, JFJ L, den Dunnen JT. LOVD v.2.0: The next generation in gene variant databases. Hum Mutat. 2011;32:1-7.

38. Huang DW, Sherman BT, Lempicki RA. Systematic and integrative analysis of large gene lists using DAVID bioinformatics resources. Nat Protoc. 2009;4:44-57.

39. Szklarczyk D, Franceschini A, Wyder S, Forslund K, Heller D, Huerta-Cepas J et al. STRING v10: protein-protein interaction networks, integrated over the tree of life. Nucleic Acids Res. 2015;43(Database issue):D447-52.

40. López-Cortés A, Paz-y-Miño C, Cabrera-Andrade A, Barigye SJ, Munteanu CR, González-Díaz H, et al. Gene prioritization, communality analysis, networking and metabolic integrated pathway to better understand breast cancer pathogenesis. Sci Rep. 2018;8:16679.

41. Beigelman A, Levy J, Hadad N, Pinsk V, Haim A, Fruchtman Y, et al. Abnormal neutrophil chemotactic activity in children with congenital insensitivity to pain with anhidrosis (CIPA): the role of nerve growth factor. Clin Immunol. 2009;365-72.

42. Indo Y, Mardy S, Tsuruta M, Karim MA, Matsuda I. Structure and organization of the human TRKA gene encoding a high affinity receptor for nerve growth factor. Jpn J Hum Genet. 1997:42:343-51.

43. Kaplan DR, Miller FD. Neurotrophin signal transduction in the nervous system. Curr Opin Neurobiol. 2000;10:381-91.

44. Wang QL, Guo S, Duan G, Ying Y, Huang P, Liu JY, et al. Phenotypes and Genotypes in Five Children with Congenital Insensitivity to Pain with Anhidrosis. Pediatr Neurol. 2016;61:63-9.

45. Ogata H, Goto S, Sato K, Fujibuchi W, Bono H, Kanehisa M. KEGG: Kyoto encyclopedia of genes and genomes. Nucleic Acids Res. 1999;27:29-34.

46. Antonov AV, Schmidt EE, Dietmann S, Krestyaninova M, Hermjakob H. R spider: a network-based analysis of gene lists by combining signaling and metabolic pathways from Reactome and KEGG databases. Nucleic Acids Res. 2010;38(Web Server issue):W78-83.

\section{Publisher's Note}

Springer Nature remains neutral with regard to jurisdictional claims in published maps and institutional affiliations. 